\title{
Factors That Affect The Severity of Dengue
}

\author{
Ruth Priya Senthiappan ${ }^{1}$ Smitha Bhat ${ }^{2}$ \\ ${ }^{I}$ Resident, Department of Medicine, Father Muller Medical College, Mangalore \\ ${ }^{2}$ Professor, Department of Medicine, Father Muller Medical College, Mangalore
}

\begin{abstract}
Dengue is the most important viral vector transmitted disease world wide in terms of morbidity and mortality. According to WHO around 5,00,000 cases of DHF(Dengue hemorrhagic Fever) and DSS(Dengue Shock Syndrome) occur annually with 22,000 deaths. It is found that some individuals have only undifferentiated fever while others progress to DHF. Our study is an attempt to find out the factors that determine the severity of dengue in our population. Among the 50 individuals with dengue fever that were studied those with hypertension, diabetes, renal failure and alcohol abuse tended to have severe dengue.
\end{abstract}

Keywords: Dengue Hemorrhagic Fever, Severity

\section{Introduction}

Dengue is the most important viral-vector transmitted disease worldwide in terms of morbidity and mortality. According to $\mathrm{WHO}$ an estimated 50 million dengue infections occur annually and approximately 2.5 billion people live in dengue endemic countries. Around 5,00,000 cases of DHF and DSS occur annually with 22,000 deaths. In India, Indonesia and Myanmar, focal outbreaks away from the urban areas have reported case fatality rates of 3-5\%. ${ }^{[1]}$ The clinical presentation and severity of dengue varies among various individuals. ${ }^{[1]}$ It is not clear what determines the severity. Predicting which individuals will suffer from a severe manifestation of dengue is crucial for early triage and appropriate intensive care. Our study is an effort to determine the various factors in our population that predispose individuals to severe dengue.

\section{Aims And Objectives of The Study}

To find if specific demographic and clinical factors predispose to severe dengue.

\section{Material And Methods}

To find out if there are factors which predispose to severe dengue. In this cross sectional analytical study, the various factors that can predispose to severe dengue were studied among 50 patients diagnosed with dengue, who were admitted to Father Muller Medical College Hospital Mangalore.

The presence of diabetes mellitus, hypertension, ischemic heart disease, stroke, bronchial asthma and renal failure were noted. Age, ethnicity and religion were documented. Exercise, diet, addiction to alcohol and nicotine were noted. An assessment of the severity of dengue is done using the dengue severity score as published in the WHO 2014 guidelines on dengue that classifies dengue into non severe dengue and severe dengue. The patients affected with dengue were divided into those with dengue fever, those with dengue fever with warning signs and those with severe dengue.

\section{Results}

Below are the factors present among those with severe dengue and dengue with warning signs:

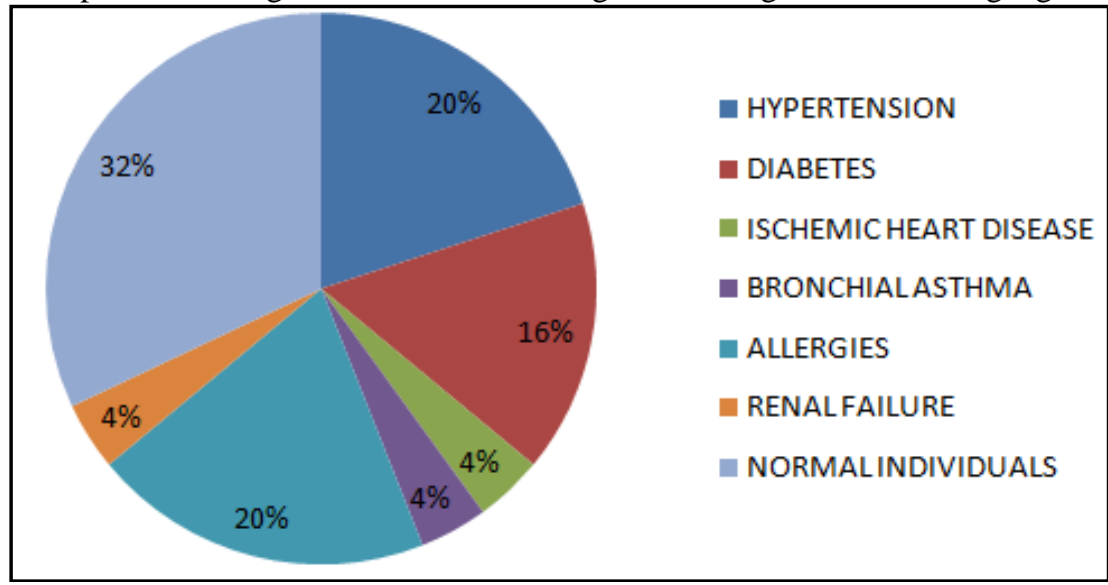




\section{Discussion}

The present WHO guidelines classifies those with dengue into 2 groups: non-severe dengue and severe dengue. Non severe dengue is again classified into those with warning signs and those without warning signs. The classification of dengue based on the level of severity is helpful in triaging patients and helps decide which patients require more close monitoring. ${ }^{[1]}$

We found that the mean age of individuals in the group with dengue fever was 43.8 years, in the group with dengue fever with warning signs was 40.54 years and in the group with severe dengue was 41.58 years. There was no significant difference in age among the groups. In a study done by Maria $\mathrm{G}$ et al. it was found that the elderly were likely to develop severe dengue-DHF and DSS. ${ }^{[2]}$ Another study done in Puerto Rica also showed that the elderly were more commonly affected with severe dengue than younger individuals. ${ }^{[3]}$ However a study done in Singapore showed that severe dengue was not associated with elderly individuals. ${ }^{[4]}$ Our study does not reflect the effect of age on the severity of dengue perhaps because very few of our patients belonged to the extremes of age.

Our study showed that those with severe dengue were more likely to have diabetes. This is similar to a study done in Kerala that showed that those with diabetes were more likely to have severe dengue. ${ }^{[5]}$ A study done by Juxiong Pang et al. in Singapore showed that individuals with diabetes had a higher risk of developing DHF. ${ }^{[6]}$ This is perhaps because diabetes is a proinflammatory state.

Our study showed that those with bronchial asthma were more likely to have severe dengue. In a study done by Bravo JR et al. it was found that bronchial asthma was associated with DHF. ${ }^{[7]}$ This is similar to a study done by Maria et al. in which it was found, that those with allergies were more likely to have DHF.A possible explanation for this is the fact that allergic individuals have signs of inflammation in the tissues and capillaries as a result of release of inflammatory cytokines especially in the endothelium. This results in increased capillary fragility and loss of third space fluid seen in severe dengue. ${ }^{[8]}$

Our study found that those with renal failure had severe dengue. In a study done in Cuba among patients with dengue fever it was found that those with chronic renal failure tended to have severe dengue. ${ }^{[9]}$ Stroke as a risk factor for severe dengue could not be studied as there were no patients with stroke in our study population.

We did not find that BMI influences the severity of dengue. The mean BMI in those with dengue fever was $22.18 \mathrm{~kg} / \mathrm{m} 2$, in those with warning signs was $22.77 \mathrm{~kg} / \mathrm{m}^{2}$ and in those with severe dengue was $22.18 \mathrm{~kg} / \mathrm{m}^{2}$. A study done by Pichainarong $\mathrm{N}$ et al. showed that obesity was associated with severe dengue. ${ }^{[10]}$ Kalayanarooj et al. studied individuals with various nutritional status and found that those with malnutrition had a greater risk of shock compared to the obese and those with normal nutritional status. The risk of encephalopathy, associated infections and complications of fluid overload were more in obese patients compared to normal and malnourished patients. The case-fatality rates in malnourished patients and obese patients were higher compared to the normal individuals. ${ }^{[11]}$ The effect of BMI in our population was not seen because most patients in this study had near normal BMI.

In our study we found that those with liver dysfunction had severe dengue. Liver injury is a positive predictive factor for the development of DHF. ${ }^{[12]}$ Chronic liver disease, alcoholic steatonecrosis, and hepatotoxic drug use such as salicylates, acetaminophen in the setting of dengue infection predisposes to and even increases liver injury ${ }^{[13]}$.In a study done among dengue patients it was found that those with alcohol consumption were less likely to develop dengue and hence concluded that chronic alcohol intake is protective against dengue infection. ${ }^{[14]}$ No correlation was found between severity of dengue and smoking,exercise, and tobacco chewing.

Reinfection with a different serotype affects the severity of dengue. A study done by Maria et al. showed that reinfection with a different serotype of dengue causes a more severe manifestation of dengue. ${ }^{[15]}$ However in a study done by Douglas et al. among Iquitos it was found that reinfection with American dengue 2 serotype was not found to cause severe dengue as compared to reinfection with Asian dengue 2 serotype which lead to DHF. ${ }^{[16]}$

Ethnicity affects the severity of dengue. Studies done have shown that Africans had a lesser predisposition to severe dengue when compared to the Caucasians. ${ }^{[17]}$ In a study done in Singapore, more Chinese patients with dengue than Indians and Malaysians, progressed to DHF. ${ }^{[8]}$

Out of the 19 individuals who had a rubber plantation near their residence $12(63 \%)$ said that individuals in their locality were also affected with dengue. Hence the rubber plantation might be responsible of the breeding of mosquitoes and hence the disease. ${ }^{[18]}$ The latex-collecting cups tethered along the main trunk of the rubber tree are breeding ground for mosquitoes. ${ }^{[19]}$ This study was limited by the fact that the sample size was small and that not many people in the sample had severe dengue as per WHO guidelines. Further research is warranted with a larger sample size. 


\section{Conclusion}

We found that individuals with diabetes, hypertension, renal failure, IHD and alcohol consumption have more severe dengue. Therefore, these individuals might benefit from more intensive care and monitoring.

\section{References}

[1] Dengue: Guidelines for Diagnosis, Treatment, Prevention and Control: New Edition. Geneva: World Health Organization; 2009.

[2] Guzmán MG, Kouri G, Bravo J, Valdes L, Vazquez S, Halstead SB. Effect of age on outcome of secondary dengue 2 infections. Int J Infect Dis. 2002 Jun;6(2):118-24.

[3] García-Rivera EJ, Rigau-Pérez JG. Dengue severity in the elderly in Puerto Rico. Rev Panam Salud Publica. 2003 Jun;13(6):362-8.

[4] Lye DC, Lee VJ, Sun Y, Leo YS. The benign nature of acute dengue infection in hospitalized older adults in Singapore. Int J Infect Dis. 2010 May; 14(5):e410-13

[5] Karunakaran A, Ilyas WM, Sheen SF, Jose NK, Nujum ZT. Risk factors of mortality among dengue patients admitted to a tertiary care setting in Kerala, India. J Infect Public Health. 2014 Mar-Apr;7(2):114-20.

[6] .Pang J, Salim A, Lee VJ, Hibberd ML, Chia KS, Leo YS, et al. Diabetes with hypertension as risk factors for adult dengue hemorrhagic fever in a predominantly dengue serotype 2 epidemic: a case control study. PLoS Negl Trop Dis. 2012;6(5).

[7] Bravo JR, Guzmán MG, Kouri GP. Why dengue haemorrhagic fever in Cuba? 1.Individual risk factors for dengue haemorrhagic fever/dengue shock syndrome (DHF/DSS). Trans R Soc Trop Med Hyg. 1987;81(5):816-20.

[8] Figueiredo MA, Rodrigues LC, Barreto ML, Lima JW, Costa MC, Morato et al. Allergies and diabetes as risk factors for dengue hemorrhagic fever: results of a case control study. PLoS Negl Trop Dis. 2010 Jun 1;4(6).

[9] Kuo M, Lu PL, Chang J, Lin M,Tsai J,Chen Y. Impact of Renal Failure on the Outcome of Dengue Viral Infection. Clin J Am Soc Nephrol. 2008; 3(5):1350-56.

[10] Pichainarong N, Mongkalangoon N, Kalayanarooj S, Chaveepojnkamjorn W. Relationship between body size and severity of dengue hemorrhagic fever amongchildren aged 0-14 years. Southeast Asian J Trop Med Public Health. 2006 Mar;37(2):283.

[11] Kalayanarooj S, Nimmannitya S. Is dengue severity related to nutritional status? Southeast Asian J Trop Med Public Health. 2005 Mar;36(2):378-84

[12] Kalayanarooj S, Vaughn DW, Nimmannitya S, Green S, Suntayakorn S, Kunentrasai N et al.Early clinical and laboratory indicators of acute dengue illness. J Infect Dis. 1997 Aug; 176(2):313-21.

[13] Mourão MP, Lacerda MV, Bastos Mde S, Albuquerque BC, Alecrim WD et al. Dengue hemorrhagic fever and acute hepatitis: a case report. Braz J Infect Dis. 2004 Dec;8(6):461-4.

[14] Pandejpong D, Saengsuri P, Rattarittamrong R, Rujipattanakul T, Chouriyagune C. Is excessive acetaminophen intake associated with transaminitis in adult patients with dengue fever? Intern Med J. 2015 Jun;45 6):653-8.

[15] Guzmán MG, Alvarez M, Rodríguez R, Rosario D, Vázquez S, Valds L, et al. Fatal dengue hemorrhagic fever in Cuba, 1997. Int J Infect Dis. 1999 Spring;3(3):130-5.

[16] Watts DM, Porter KR, Putvatana P, Vasquez B, Calampa C, Hayes CG, et al Failure of secondary infection with American genotype dengue 2 to cause dengue haemorrhagic fever. Lancet. 1999 Oct 23;354(9188):1431-4.

[17] de la C Sierra B, Kourí G, Guzmán MG. Race: a risk factor for dengue hemorrhagic fever. Arch Virol. 2007;152(3):533-42.

[18] Sumodan, P. K. Potential of rubber plantations as breeding source for Aedes albopictus in Kerala, India. Dengue Bull 27: $197,2003$.

[19] Dengue in Kerala :A critical review.ICMR Bull 36:4-5,2006. 